\title{
lomt Ecosystem for Secured Woman Health Monitoring System (WHMS)
}

\author{
Rasika Mallya \\ MCA, DES's NMITD, Mumbai, India
}

\section{ABSTRACT}

An Internet of Medical Things is a system with network of medical devices and people to implement ubiquitous healthcare or assistive healthcare system. This paper applies secure MQTT communication protocol to Woman Health Monitoring System (WHMS) to ensure security of machine to machine (M2M) communication. Woman Health Monitoring System is assistive healthcare system for any woman who can take care of her health with this monitoring system. This paper represents how the secured communication occurs with integration of MQTT frames in software agents of Woman Health Monitoring System.

\section{KEY WORDS: IOMT, WOMAN HEALTH MONITORING SYSTEM, SECURE MQTT, M2M COMMUNICATION.}

\section{INTRODUCTION}

An Internet of Medical Things (IoMT) ecosystem comprises of connected medical devices, software applications, health services to generate, collect, analyze or transmit health data or images and connect to healthcare provider to streamline clinical workflow with the aim of improving patient care, both inside care facility walls and in remote locations. Remote health monitoring system with various sensors allow real-time monitoring of different body parameters including BP, sugar level, stress level, movements, fall monitoring which help for prevention of serious illness and timely treatment to remote patients or senior citizens. Such ubiquitous healthcare systems play vital role in telemonitoring, digital diagnostics as well as they create awareness among people about self-care preventing frequent visits to hospitals. This approach is extended for any age women-specially in Indian Society.

Biosc Biotech Res Comm P-ISSN: 0974-6455 E-ISSN: 2321-4007

\section{crossef}

Identifiers and Pagination

Year: 2021 Vol: 14 No (5) Special Issue

Pages: 74-79

This is an open access article under Creative

Commons License Attribn 4.0 Intl (CC-BY).

DOI: $h t t p: / / d x$. doi.org/10.21786/bbrc/14.5/15
Indian women avoid to visit physicians till they face serious illness neglecting minute alarming situations of health issues.

Using the concept of remote patient monitoring, Woman Health Monitoring System has been proposed. In this system, woman of any age can use specific wearables to monitor regular body parameters like pulse rate, heart rate, blood-sugar level, body temperature, workout monitoring etc. The proposed system also covers ovulation monitoring, fetus monitoring for women who are either under the treatment of infertility or women are pregnant. This paper applies concept of Internet of Medical Things (IoMT) with MQTT protocol to build secured ecosystem for WHMS. AUMLis applied to generate agent interaction protocols to represent pattern of communication among components of MQTT protocol.

Section 2 will define various components of IoMT along with bio-sensors and MQTT protocol. Section 3 describes IoMT ecosystem for WHMS. Section 4 represents agent interaction protocol for MQTT protocol applied for WHMS. Section 5 concludes the work.

\section{Literature Review:}

2.1 Internet of Medical Things (IoMT): IoMT is the complicated, interconnected structure of all devices used for health monitoring and streamlining health services.

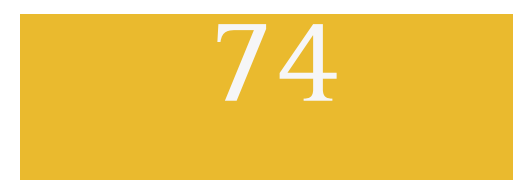


IoMT devices with the support of artificial intelligence techniques form intelligent system. This ubiquitous and intelligent system can monitor all body parameters for remote treatment as well as provides alert and alarm system to caregiver of patient and concerned physician. IoMT-driven systems provide right treatment to right patient at any place, at any time. IoMT ecosystem mainly comprises of three components: Data acquisition, communication gateway and Cloud storage. Figure 1 shows architecture of IoMT ecosystem.

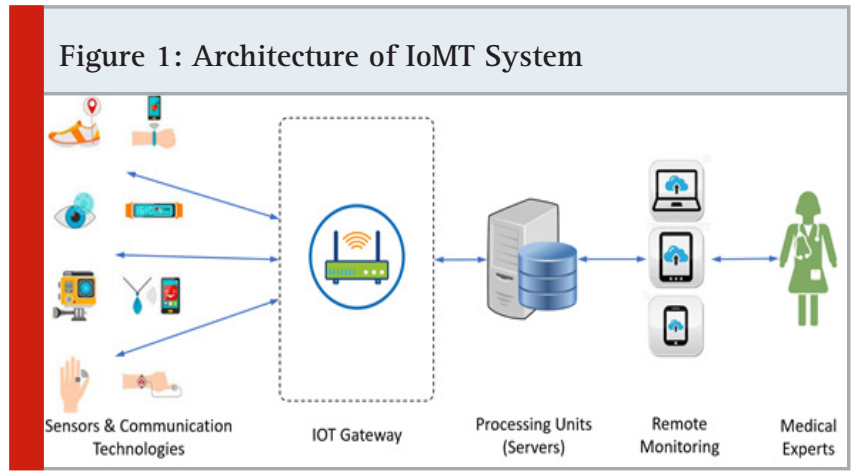

Figure 2: IoMT Components
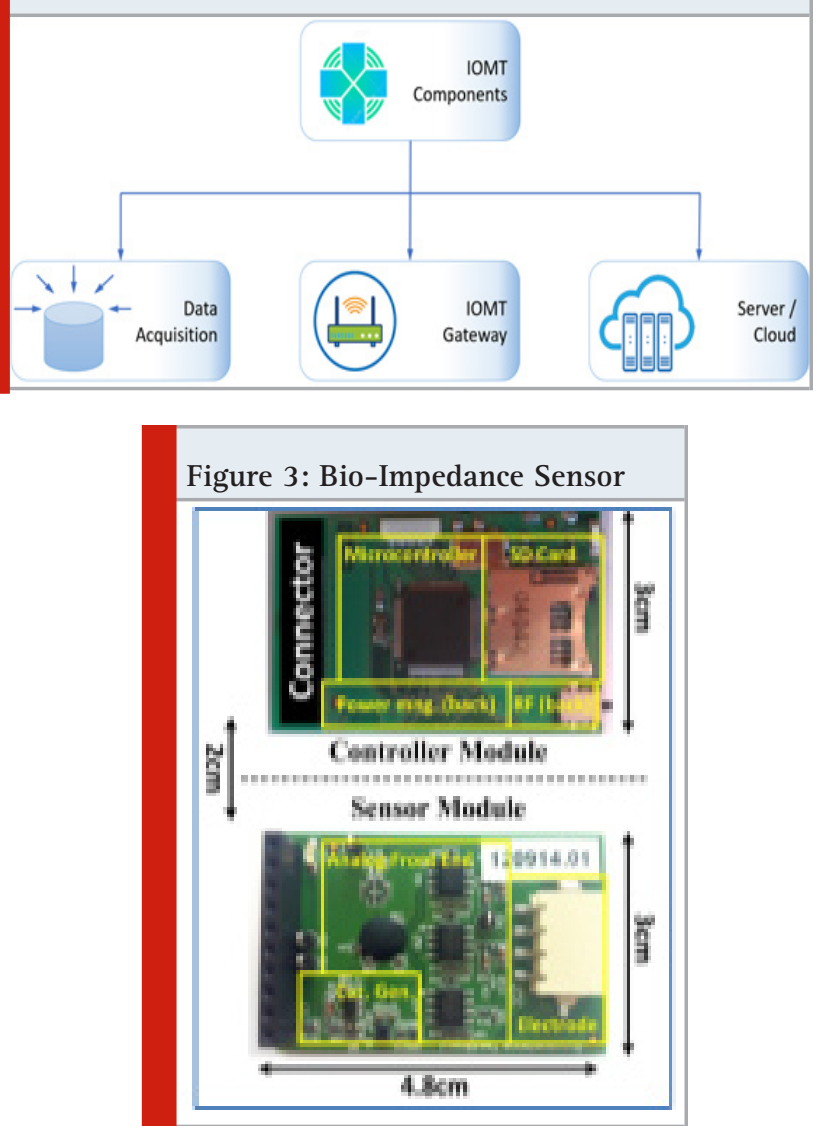

Figure 2 shows components of IoMT. Data acquisition occurs through sensors/wearables of system. After preprocessing of data, data is sent to cloud storage through communication gateways. At cloud data is used for further analysis and specific indicators are generated for patient's health.
2.1.1 Data Acquisition in IoMT System: In IoMT ecosystem, first important task is monitoring body parameters of patient/ user, preprocess it, remove noise and send it to cloud storage. For monitoring body parameters, bio-sensors are used. The bio-sensors are electronic devices. These devices capture body parameters functioning for specific time interval, preprocess them and send to caregivers. Some bio-sensors have the capability to generate alert/ alarm if monitored data is presenting abnormal patterns. They are classified in two ways: Wearable sensors and non-wearable sensors.

2.1.2 Wearable Sensors: Wearable sensors are smart sensing devices with good sensing capability, are wore on body. They function without human intervention to capture body parameters. Some of the wearable sensors are mentioned below:

a. Bio-impedance Sensor: It can be used to measure respiration rate, heart rate and cardiovascular pressure (CVP). It consists of sensor and controllable module as shown in figure 6.6. Hardware configurations of bioimpedance sensor module is it is with microcontroller, radio with frequency $2.4 \mathrm{GHz}$ and micro SD.

b. Accelerometer: Accelerometer is used to detect fall of a user. It can be also used as heart and activity monitor. An accelerometer branded by Alive Technologies is shown in fig 4.This device is used to monitor exact position of the user, speed of activity performed by the user, ECG and heart rate in real time along with fall detection. This device is famous for cardiac rehabilitation programs and monitoring performance of athletes.

Figure 4: Accelerometer

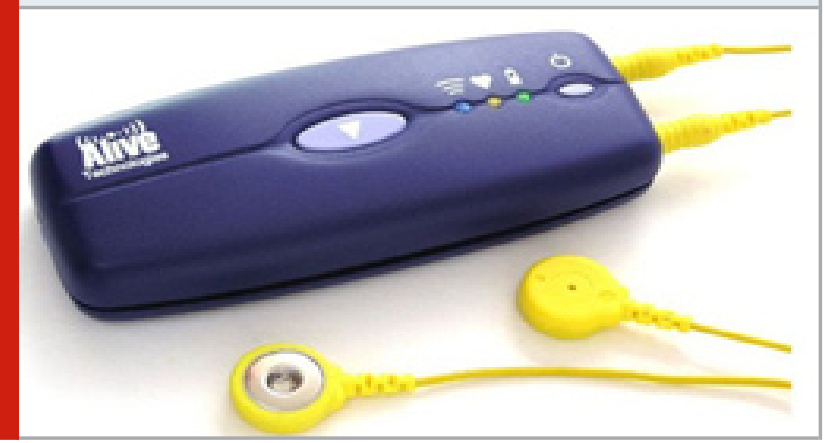

Figure 5: Photoplethysmograph Device

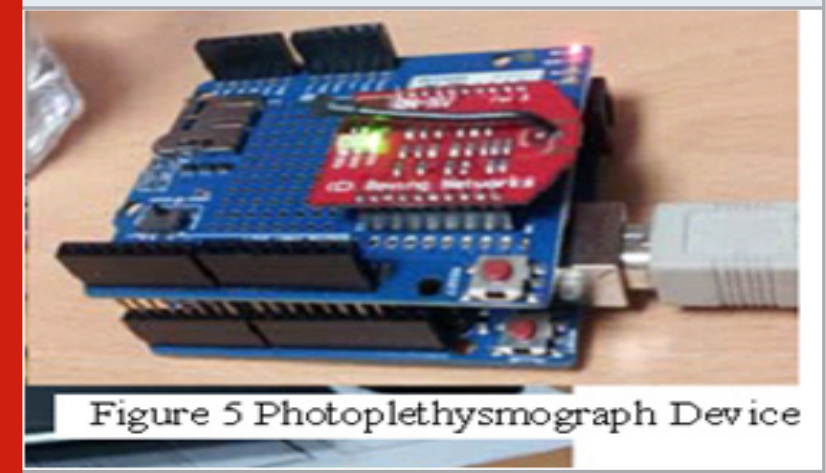


c. Photoplethysmograph: The Photoplethysmograph is used to measure heart rate variability (HRV) calculations, pulse rate, breathing rate, and perfusion measurements. It is optical health monitoring sensor. Hardware configuration: rechargeable Li-Ion accumulator with voltage stabilizers, connected to central processor, Bluetooth module and analogue circuit, infrared LED, works on USART (Universal Synchronous Asynchronous Receiver/Transmitter) protocol.

d. Pulse Oximeter: Pulse oximetry sensors or $\mathrm{SpO} 2$ monitor is a wearable device used to determine person's blood oxygen saturation. It is an important tool to monitor respiratory functions of any individual. A wearable Sp02 monitor is shown in Fig. 6. There are two factors in human blood such as oxygenated hemoglobin $\mathrm{Hb}$ and deoxygenated hemoglobin (deoxy-Hb), which are used to measure human blood oxygen level. Important components of oximeter are optical transmitter, receiver, microcontroller, PCB, Bluetooth communication. Heart rate algorithm is used to calculate heart rate after specific interval. Similar to oximeter, the other wearable, named as pulse rate monitor is used to monitor ECG signals, blood pressure, body heat, and respiration. It uses arduino as interface between pulse sensor and computer.

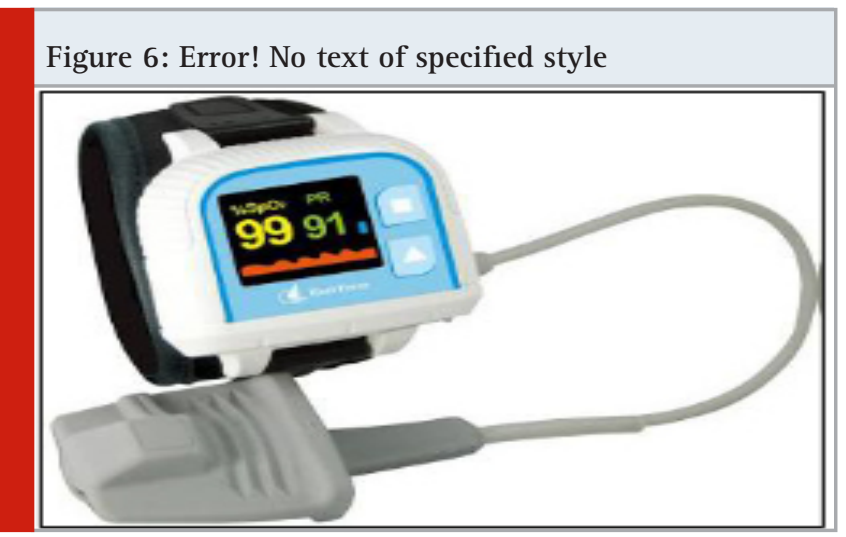

2.1.1.1 Non-Wearable Sensors: Non-wearable sensors are also smart sensing devices but cannot be worn on human body and work without human intervnetion. They are fixed in surrounding of human body to monitor activities of patient/user. Some of the non-wearable sensors are mentioned below:

a. Smart Beds: These sensors permit monitoring of patient remotely without touching the patient. Smart beds have sensors imbibed with them to monitor respiration, temperature, sleep pattern etc. The beds have alarm functionality also. These smart beds are useful in special cases of pandemic where touching patient can be dangerous for caregivers/ health practitioners.

b. Human Activity Detection: This function is implemented with different WSNs and CCTVs encorporated in home where the patient stays. The caregiver can watch these activities remotely for further analysis or safety. This functionality is specially useful in treatment of cardiovascular disorders, stroke, senior citizens care or parkinsons etc.
2.1.2 loMT Gateways: The smart devices dont have capability to transfer data to server, so IoMT gateways are physical devices or software programs which connect bionsensors with remaining IoMT application. The role of gateway is normalization of data and network connectivity. During normalizing captured data, gateways convert data from different formats into unique format which is preprocessed further for analysis. Through network connectivity, the bio-sensors are connected to WAN for communication.

2.1.3 Cloud Storage: The servers are part of cloud storage device of IoMT, which is core part of complete system responsible for decision of diagnostics and prescribing further treatment based on monitored data. They implement signal processing techniques and covert data into desired format, data mining algorithms for right predictions, neural networks for discovering patterns of monitored data to recognize any abnormal pattern. In this way, IoMT creates the ecosystem for remote patient monitoring.

\subsection{Woman Health Monitoring System:}

Figure 7: Design View of Woman Health Monitoring System

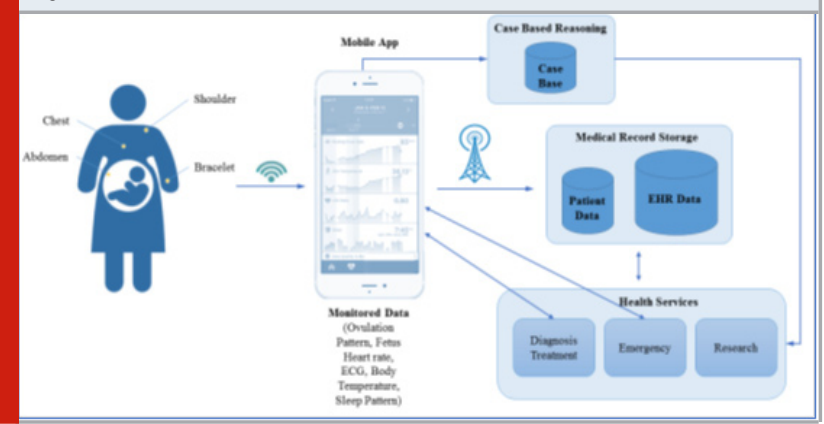

The main goal of Woman Health Monitoring System is monitoring body parameters of woman of any age to make her aware about her own health so she can take good care of her own health and can visit physician when she observes any abnormal body parameters or alarms in her smart monitoring system. Following types of monitoring are possible with WHMS: i) Ovulation monitoring if the woman wants to conceive and she is under treatment of obstetrician ii) Fetus monitoring is the woman is pregnant iii) Menopause monitoring if the woman is facing hormonal imbalance problem iv) Regular body parameters like pulse rate, heart rate, blood-sugar level, body temperature, workout monitoring etc. Consider the WHMS as shown in figure 7 . The smart devices are connected with mobile app where monitored raw data will be sent for storage as well as further diagnosis. If the monitoring data shows abnormal changes in parameters, the system generates alerts for physician and home caretaker.

If the situation is controlled situation then physician provides treatment through telecalling else she has been advised to see physician as early as possible. Here, the system depends on two databases: Electronic Health 
Record database which maintains important medical history, the second database is patient's recent data captured by bio-sensors. The app is connected with health service provider which will diagnose change in body parameters. The monitored data, treatment and line of prescription will be made available for different health services like i) Diagnosis and treatment by concerned physician ii) The possibility of emergency can be detected by stored pattern.

2.3 MOTT Communication Protocol: The proposed WHMS can be made more secured and robust with integration of MQTT Communication Protocol. The MQ Telemetry Transport (MQTT) protocol, described by OASIS is communication protocol for IoT applications. It provides one-to-many message distribution based on publish/subscribe messaging protocol. This protocol is used for machine-to-machine communication where one or many machines are nominated as clients i.e. end devices responsible for generating data and servers are responsible for collating monitored data and converting it into useful information for IoT Application. This protocol supports three types of communication. Each type of communication is identified as QoS in this context and those are described as follows: Client -> Server : Publish Server Action : Publish message to subscribers

Table 1: MQTT QoS $=0$ Protocol

Table 1. Represents simple MQTT message with QoS=0 which is simple One client to multiple receiving devices communication.

\begin{tabular}{|l|c|}
\hline Client $->$ Server : & Publish \\
\hline Client Action : & Store Message \\
\hline Server Actions : & Store Message; \\
\hline $\begin{array}{l}\text { Publish message to } \\
\text { subscribers; }\end{array}$ & Delete Message \\
\hline Server -> Client : & Puback \\
\hline Client Action : & Discard Message \\
\hline
\end{tabular}

This protocol ensures that the published message is guaranteed to arrive only once at the subscribers. In this protocol, Pubrec and Pubcomp represent acknowledgment messages from the server, whereas Pubrel is an acknowledgment message from the client. The loss of Pubrec causes the client to re-execute the protocol from its beginning, whereas the loss of Pubcomp causes the client to retransmit only the second part of the protocol, which starts at the Pubrel message. This additional machinery presumably ensures a single delivery of the published message to the subscribers. The message format handled by MQTT is as follows:

As shown in figure 8, MQTT header contains field message type which indicates the message is from sender or publisher, variable header contains username and password flag which can be used for authentication of message. The MQTT protocol requires three entities for execution: i. Publisher entity to publish data ii.
Subscriber entity receives the data through a Broker. Iii. PKG or broker is the trusted third party as facilitator. There are four phases in the protocol. Setup phase is responsible for registration and key management. Data is encrypted in encrypt phase and in publish phase, Publisher publishes encrypted data and sends it to the broker. In decrypt phase, subscribed devices decrypt data. Figure 9 represents sequence diagram of SecureMQTT.

Table 2. MQTT QoS = 1 Protocol

\begin{tabular}{l|c}
\hline Client -> Server : & Publish \\
Client Action : & Store Message \\
Server Actions : & Store Message OR \\
Publish message & Store Message ID; \\
to subscribers & \\
Server -> Client : & Pubrec \\
Client -> Server : & Pubrel \\
Server Actions : & Publish message to \\
& subscribers; Delete Message \\
Server $->$ Client : & OR Delete Message ID \\
Client Action : & Pubcomp \\
\hline
\end{tabular}

Figure 8: MQTT Header Frame Format

\begin{tabular}{|c|c|c|c|c|c|c|}
\hline bit & 7 & 6 & \begin{tabular}{l|l}
5 \\
\end{tabular} & 3 & 2 & 0 \\
\hline byte 1 & & lessa & Type & DUP Flag & QoS Level & Ret \\
\hline byte 2 & \multicolumn{6}{|c|}{ Remaining Length } \\
\hline \multicolumn{7}{|c|}{ Variable Header } \\
\hline \multicolumn{7}{|c|}{ Payload } \\
\hline
\end{tabular}

1. Publisher and receiver devices register with Broker (Third party element) for secured data transfer.

2. Publisher device generates access tree based on access policy

3. Publisher device encrypts data using public parameters and generates ciphertext

4. Publisher embeds encrypted data in SPublish and sends It

5. Broker responds with PUBACK packet.

6. Publisher sends PUBREL packet to Broker.

7. Broker deletes data and sends PUBCOMP packet to Sender.

In this way, publishing device and receiving device communicate with each other in secured enviornment. The next section represents MQTT protocol in more secured way specially for sensor networks.

III. WHMS WITH INTEGRATED SMOTT: MQTT integration for any IoT application is always significant due to nature of MQTT. When MQTT is compared with COAP, XMPP, it has been found that MQTT needs low battery consumption, less utilization of CPU and network and mainly its simplicity for implementation. With Secure MQTT, it is possible to implement encryption and secure delivery among multiple communication nodes. As shown in figure 10, WHMS will communicate with its 
stakeholders after integration of MQTT. After integration of MQTT, WHMS gets functionalities such as

1. Technical alarms as well as clinical alarms

2. Internally, prioritization among messages without human intervention

3. Secured environment of message passing through encryption-decryption of MQTT.

Figure 9: Sequence Diagram of Secure-MQTT

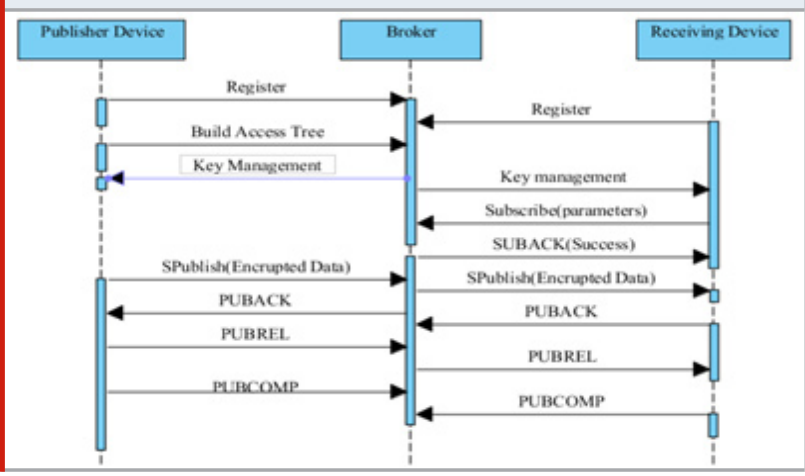

Figure 10: WHMS with S-MQTT Block Diagram

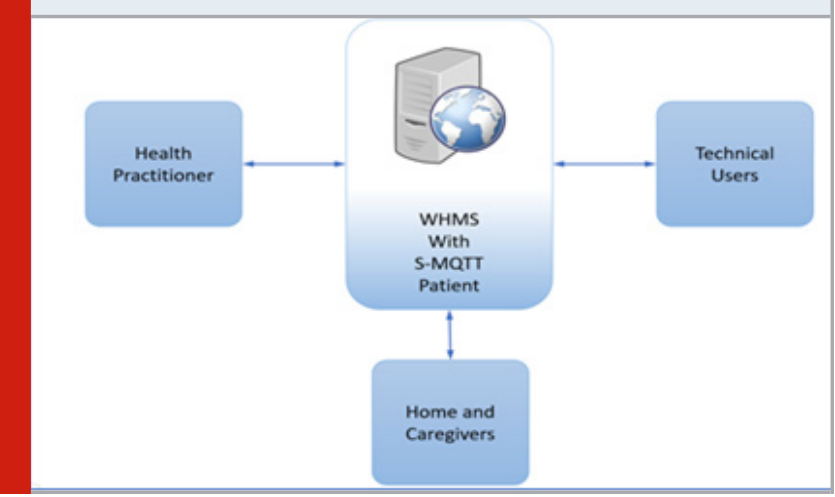

Following scenario explains how MOTT functions in WHMS: The scenario assumes that the pregnant lady is using CTG sensor to monitor her fetus movements and breathing rate. As shown in figure 11 represents architecture for given scenario where publisher device is CTG sensor and there are multiple receiving devices as mobile agent of user and mobile agent of gynecologist. The publishing and receiving devices have the artifcats for encryption and decryption of messages. The curved lines in figure represents UDP connection with broker.

Following sequence diagram (figure 12) describes how publishing device i.e. CTG sensor communicates with receiving devices like user and gynecologist as and when required based on scenario. Message numbers 8 and 9 show that when fetus body parameters are normal, the messages are sent to only one receiving device i.e. user. But when less fetus movements are detected by CTG sensor, the SPublish message is sent to Gynecologist receiving device also along with user receiving device. The controlling component of WHMS will instruct broker to send SPublish frame to gynecologist in terms of clinical alarm. Figure 11 uses formats of agent interaction protocol which is depicted through sequence diagram. Each lifeline is sequence diagram represents agent in communication.

Figure 11: MQTT-SN Architecture for Scenario

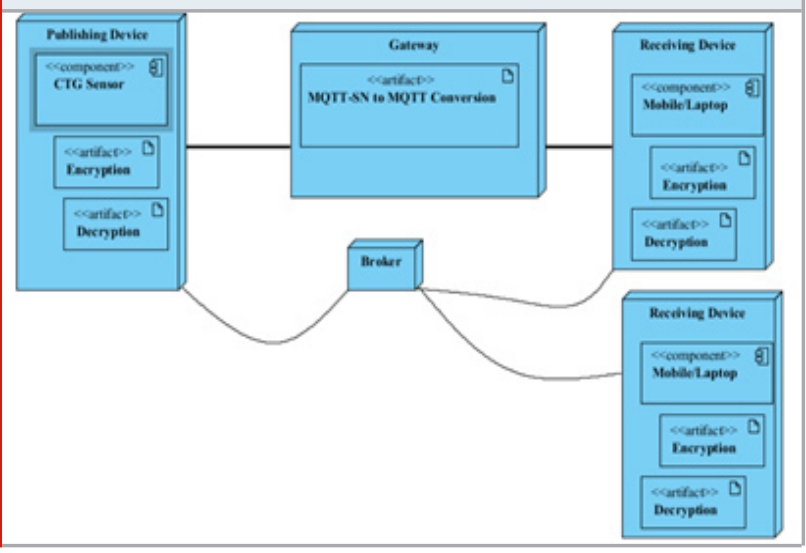

Figure 12: MQTT-SN Protocol Implementation with CTG Sensor as publishing device

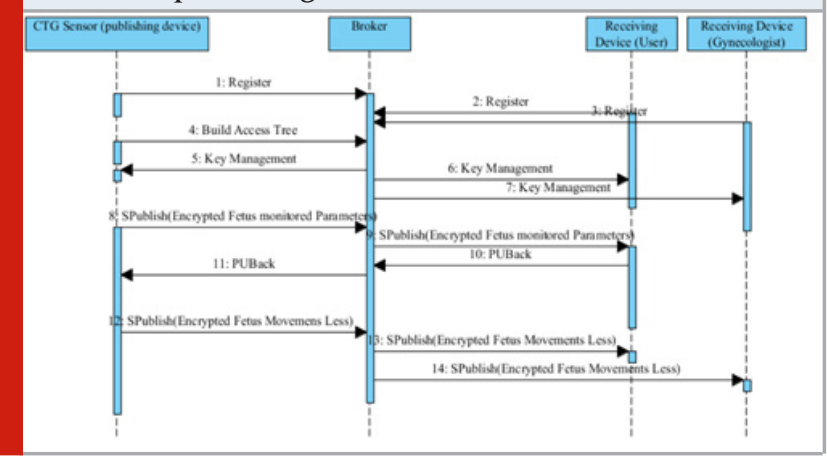

As described in this scenario, MQTT protocol can handle heterogeneity in various scenarios and actors in accordance with multiple publishing devices and receiving devices. Figure 11 also describes that CTG sensor is at main focus since it is publishing device and other actors like user or gynecologist are playing passive roles, receiving information from user, based on needs, authorization and consent of user.

\section{CONCLUSION}

This paper represents study of Internet of Medical Things which is in much-demand to streamline healthcare services without increasing burden on health practitioners. As mentioned in study, IoMT supports remote healthcare practices with bio-sensors, gateways and cloud support. This paper also explores communication protocol - MQTT which is lightweight messaging protocol with pub/sub patterns. In this study, MQTT is applied for Woman Health Monitoring System which is Remote Healthcare system for women. The implementation section of this paper represents how MQTT protocol generates messaging frames in controlled manner for specific receiving devices and it can take decision autonomously to include new receiving device in communication as per the scenario. With application 
of Secure MQTT protocol, WHMS becomes more secured and controlled.

\section{REFERENCES}

https://healthtechmagazine.net/article/2020/01/ how-internet-medical-things-impacting-healthcareperfcon

David Naranjo-Hernández, IAlejandro TalaminosBarroso , Javier Reina-Tosina , Laura M. Roa, Gerardo Barbarov-Rostan, Pilar Cejudo-Ramos, Eduardo Márquez-Martín and Francisco Ortega-Ruiz, "Smart Vest for Respiratory Rate Monitoring of COPD Patients Based on Non-Contact Capacitive Sensing", Sensors 2018, 18, 2144; doi:10.3390/s18072144, www.mdpi.com/journal/ sensors

R. Mallya, S. Kothari, "Requirements model for adaptive information system for woman health monitoring system," 2018 International Conference on Communication information and Computing Technology (ICCICT), Mumbai, 2018, pp. 1-6, doi: 10.1109/ICCICT.2018.8325882.

Fadi Al-Turjman, Muhammad Hassan Nawaz, Umit Deniz Ulusar, "Intelligence in the Internet of Medical Things era: A systematic review of current and future trends", Computer Communications 150 (2020) 644660, https://doi.org/10.1016/j.comcom.2019.12.030, 0140-3664/๑ 2019 Elsevier B.V.

Seulki Lee et al, "A Low-power and Compact-sized Wearable Bio-impedance Monitor with Wireless Connectivity”, 2013 J. Phys.: Conf. Ser. 434012013. https://www.avawomen.com/how-ava-works/ healthcare/technology/\#conceiving

https://www.alivetec.com/pages/alive-bluetooth-heartactivity-monitor

Valerie GAY, Peter LEIJDEKKERS, "A Health Monitoring System Using Smart Phones and Wearable Sensors", International Journal of ARM, VOL. 8, NO. 2, June 2007.

Janis Spigulis, Renars Erts, Vladimirs Nikiforovs and Edgars Kviesis-Kipge, "Wearable wireless photoplethysmography sensors", Biophotonics: Photonic Solutions for Better Health Care, edited by Jürgen Popp, Wolfgang Drexler, Valery V. Tuchin, Dennis L. Matthews, Proc. of SPIE Vol. 6991, 699120, (2008) • 1605-7422/08/\$18 • doi: 10.1117/12.801966 Yogita Bobade, Prof. R. M. Walli, "A Review of Wearable Health Monitoring Systems”, International Journal of Advanced Research in Electrical, Electronics and Instrumentation Engineering (An ISO 3297: 2007 Certified Organization) Vol. 4, Issue 10, October 2015. Fanpeng Kong, Yubo Qin, Zhengyu Yang, Zhongtian Lin, "A Wearable Pulse Oximeter".

Hermawan Kemis, Ndibanje Bruce, Hoon Jae Lee, "On Pulse Sensor based u-Healthcare Monitoring Application with Arduino", Journal of Next Generation Information Technology(JNIT), Volume 4, Number 9, November 2013.

B. Aziz, "A Formal Model and Analysis of the MQ Telemetry Transport Protocol," 2014 Ninth International Conference on Availability, Reliability and Security, Fribourg, Switzerland, 2014, pp. 59-68, doi: 10.1109/ ARES.2014.15.

Meena Singh, Rajan MA, Shivraj VL, and Balamuralidhar P, “Secure MQTT for Internet of Things (IoT)", 2015 Fifth International Conference on Communication Systems and Network Technologies

Vedha N S1, Prof. B. Naveen Kumar, "IOT based Smart Device Management using MQTT Services", International Journal for Research in Applied Science \& Engineering Technology (IJRASET), ISSN: 2321-9653; IC Value: 45.98; SJ Impact Factor: 7.177, Volume 7 Issue VI, June 2019- www.jiraset.com

Mallya R., Kothari S. (2019) Agent Interaction Protocols for Fetus Monitoring System. In: Saini H., Sayal R., Govardhan A., Buyya R. (eds) Innovations in Computer Science and Engineering. Lecture Notes in Networks and Systems, vol 32. Springer, Singapore. https://doi. org/10.1007/978-981-10-8201-6_42 